\title{
Schweizer Psychiatrie im 19. Jahrhundert
}

\author{
Von Hans H. Walser
}

\section{Das «Irrenwesen» in den ersten Jahrzehnten des 19. Jahrhunderts und die ersten Reformbestrebungen}

Unter dem Einfluß der Aufklärung erwachte gegen Ende des 18. Jahrhunderts an verschiedenen Orten der Wille, für die bisher ärztlich vernachlässigten und oft unmenschlich behandelten Geisteskranken etwas zu tun. So haben - um nur die bedeutendsten Namen zu nennen - Vincenzo Chiarugi (nach 1788) in Italien, William Tuke (1796) in England, J. G. Langermann (1805) in Deutschland einen humanen Umgang mit den bedauernswerten Kranken durchgesetzt. Am berühmtesten von allen Reformern dieser Zeit ist Philipp Pinel (1745-1826) geworden, der 1793 das Hôpital Bicêtre, zwei Jahre später das Hôpital Salpêtrière in Paris übernahm. In einer gewissen Vereinfachung seiner Bestrebungen spricht man davon, daß er «den Irren die Ketten abnähme». Hier lag also ein «dramatischer Akt» vor, bei welchem man die Reform des «Irrenwesens » beginnen lassen kann. Pinel hat aber auch der wissenschaftlichen Psychiatrie neue Impulse gegeben, namentlich durch sein Buch aus dem Jahre 1801. Etwas später trat innerhalb seiner Schule auch die psychiatrische Lehrtätigkeit dazu (ab 1817 durch seinen Schüler J. E. D. Esquirol) (1).

Ein ähnlicher «dramatischer Akt» aus dem Beginn der psychiatrischen Reformbewegung fehlt in der Schweiz. Nicht, daß man die Dringlichkeit der humanitären Erfordernisse verkannt hätte: So wird berichtet, daß Aвraham Joly in Genf bereits im Jahre 1787 die Geisteskranken von ihren Ketten befreite. Auch wissenschaftliche Beiträge fehlen nicht ganz. So hat etwa der Genfer André Matthey, ein Schüler Pinels, 1816 seine Nouvelles recherches sur les maladies de l'esprit herausgegeben (29). Doch blieben diese und andere Anläufe selbst in Genf, dem medizinisch fortschrittlichsten Kanton der damaligen Schweiz, ohne größeren Widerhall.

Wesentlich erschwert wurde die Reform durch die politischen Gegebenheiten der Schweiz: der lockere Staatenbund von Kleinstaaten erschwerte eine großzügige Gesundheitspolitik und namentlich den Bau von Spitälern, so daß man in der Schweiz nach 1800 im allgemeinen noch bei den Einrichtungen aus dem vergangenen Jahrhundert blieb. Sie wurden aber ziemlich kritisiert, am erfolgreichsten durch Johann Matthias Hunger- 
BÜHLER (1805-1884). Hungerbühler war Politiker, er gehörte der katholischen, liberalen Richtung an und war in den Jahren 1838-1878 wiederholt Regierungsrat des Kantons St. Gallen. 1846 schrieb er u. a.:

«Die Morgenröthe der Befreiung der Wahnsinnigen aus der doppelten Knechtschaft, der inneren und der äußeren, ist im Allgemeinen bei uns noch nicht erschienen. Noch hat der Staat bereits in den meisten Kantonen sich dieser unglücklichen, armen Menschenklasse nicht angenommen. Eingesperrt in scheußlichen Räumen, schlechter als oft die Ställe, in welchen das liebe Vieh gehegt und gepflegt wird, auf einem Haufen Stroh, nackend, niemals erquickt durch den Genuß der reinen Luft kann man heute noch in einzelnen Kantonen unglückliche Geisteskranke finden, um die sich weder die Regierung, noch die Sanitäts-, die Polizeiund Vormundschaftsbehörden bekümmern!» (23).

Hungerbühler geht dann den Zustand des «Irrenwesens» in unserem Lande kritisch durch. Den primitivsten Zustand, bei welchem sich der Staat überhaupt nicht um die Geisteskranken kümmert, fand er noch in elf Kantonen mit rund 600000 Einwohnern, also bei fast einem Drittel der schweizerischen Bevölkerung. Es waren meist kleine, ländliche Kantone, die keine eigenen Spitäler hatten. Es kam hier noch vor, daß arme Geisteskranke an den Mindestnehmenden «abgesteigert» wurden. Besser waren die Verhältnisse in Kantonen mit größeren Städten, da es dort von alters her Hospitäler gab, die auch Geisteskranke beherbergen konnten:

Basel brachte seine Geisteskranken im sogenannten «Almosen», einem Gebäude des früheren Franziskanerklosters bei der Barfüßerkirche, unter, zusammen mit Trunkenbolden, sittlich verkommenen Individuen und chronischen Kranken (49). Erst als im Jahre 1832 Friedrich Brenner (1809-1874) leitender Arzt geworden war, besserten sich die Zustände $(5,6) .1842$ konnte Brenner mit seinen Patienten einen Neubau auf dem Markgräflerhof beziehen. Es war ein Zellenbau, der bereits nicht mehr den neuesten Forderungen entsprach, so daß C. G. JUNG der Ältere meinte, die Anlage sei schon ursprünglich verpfuscht gewesen (7). Sie leistete aber gute Dienste, besonders da von 1855 an ausgesprochene Pflegefälle in das Absonderungshaus an der Spitalgasse verlegt werden konnten. Über den lange Zeit vergessenen Friedrich Brenner und über seine Tätigkeit für die Schweizer Psychiatrie weiß man heute wieder gut Bescheid (33).

In Bern bestand weiter das alte «Tollhaus» von 1749 , das räumlich vom Inselspital getrennt war. Aber nicht nur das Gebäude, auch der Umgang 
mit den Geisteskranken war antiquiert, und, wie A. Tribolet sagt, schlechterdings «erbärmlich», bis von 1835 an bauliche Verbesserungen und gleichzeitig auch eine mildere Behandlung eingeführt wurden. Im Jahre 1855 wurde ein moderner Bau, die Waldau, eröffnet, womit Bern hinsichtlich Irrenwesen an eine der vordersten Stellen in der Schweiz vorrückte (28, $36,47^{1}$.

In Genf waren die Geisteskranken zu Beginn des Jahrhunderts in der «Discipline», einem vom Spital abhängigen Hause, untergebracht, das wie andernorts auch Kriminelle beherbergte. Der Leiter, Gaspard DE LA Rive (1770-1834), forderte vergeblich eine spezielle Abteilung für agitierte Kranke sowie ein Asyl außerhalb der Stadt. Immerhin wurden die Geisteskranken $1832 \mathrm{im}$ Schloß Corsier untergebracht, bis 1838 eine moderne Irrenanstalt, das «Asyle des Vernets», eröffnet wurde, das in JEAN CHARLES Coindet (1796-1876) einen bedeutenden Leiter erhielt. Joseph Guislain, der dieses Spital kurz nach seiner Einweihung besuchte, rühmte es als eines der besten : «... je crois devoir ranger l'Hôpital des Aliénés de Genève parmi ceux qui, au moment actuel, renferment le plus de dispositions favorables auch bien-être des malades» (18).

Über Lausanne hatte Guislain dagegen wenig Gutes zu berichten; immerhin, eine Irrenanstalt war vorhanden und eine bessere wurde angestrebt.

Zürich hatte auf dem Areal des Spitals ein Irrenhaus erbaut, doch wurde auch nachher die «Lochkammer» im nahen Spital belegt. 1842, nach dem Neubau des Spitals an einem neuen Standort, wurde das alte Spital für Geisteskranke reserviert. Hier hat zwanzig Jahre später Wilhelm GrieSINGER (1817-1868) noch gewirkt - zu einer Zeit, da die Einrichtungen veraltet und schon fast skandalös geworden waren, worüber die Ärzte August Zinn (1825-1897) sowie Hans Ulrich Zehnder (1798-1877) berichtet haben. Zehnder war wie Hungerbühler Regierungsrat, hatte sich also ex officio mit dem «Irrenwesen» zu befassen, doch erhält man den Eindruck, daß ihm dieses eine Herzensangelegenheit war. Er arbeitete insbesondere für die Einrichtungen der Anstalten Rheinau und Burghölzli, bei deren Eröffnung (1867 bzw. 1870; er allerdings nicht mehr im Amte war $(4,22,41)$.

Diese Beispiele aus den fortschrittlicheren Kantonen zeigen, daß die öffentliche Sorge für die Geisteskranken im ersten Drittel des Jahrhunderts völlig ungenügend und veraltet war. Soweit es überhaupt Irrenanstalten gab, waren sie mit den alten Spitälern organisatorisch und meist auch räumlich aufs engste verbunden. 
Privatanstalten waren zwar vorhanden, doch waren sie meist klein und oft an die Person eines Arztes gebunden, zudem konnten sie nur einigermaßen begüterte Patienten aufnehmen. Die Hauptschwierigkeit lag aber gerade bei den armen Geisteskranken. Vermögliche Geisteskranke wurden deshalb auch gerne im Ausland untergebracht, solche aus dem Kanton Neuenburg etwa in Dôle oder in Stephansfeld (Elsaß), solche aus dem Tessin in der Lombardei. Irgendwelche gesetzliche Vorschriften fehlten in der Schweiz meist noch, so daß der Staat keine Möglichkeit der Kontrolle hatte. Die ersten «Irrengesetze» sollen diejenigen von Genf (1838) und Neuenburg (1843) gewesen sein (24b). Mißbräuchen waren also Tür und Tor geöffnet, zumal wenn eine Anstalt mehr dem Erwerb als der Heilung der Kranken diente. Immerhin darf man annehmen, daß einzelne Anstalten doch in echt humanitärem Geist geführt wurden. Eine dieser Anstalten genoß sogar eine Zeitlang europäischen Ruf und ist deshalb zu erwähnen, obwohl sie erst gegen Ende dieses Zeitabschnittes (1841) eröffnet wurde und für die Patienten mit einer Krankheit bestimmt war, die nur teilweise in das Gebiet der Psychiatrie fällt: Es war der «Abendberg» bei Thun, wo der Arzt Јаков Guggenв ÜнL (1816-1863) auf vorbildliche Weise Kretinen behandelte und schulte (23).

\section{Die Schaffung unabhängiger psychiatrischer Anstalten}

Das Ziel, die psychiatrische Anstalt nicht nur von den Versorgungs- und Armenhäusern, sondern auch von den Spitälern für körperlich Kranke zu trennen, wurde etwa von der Jahrhundertmitte an in immer zunehmendem Maße erfüllt und schließlich gänzlich durchgeführt. Die Abtrennung sollte vollständig sein, also nicht nur Betrieb und ärztliche Versorgung betreffend, sondern möglichst auch einen räumlichen Abstand schaffen. So wurden den Anstalten entweder separate Gebäude (aufgehobene Klöster) überwiesen, oder es wurde ein Neubau außerhalb der Stadt errichtet. Diese Anstaltsgründungen waren: Genf 1838, Münsterlingen (Thurgau) 1840(44), Préfargier (Neuchâtel) 1848, Pirminsberg (St. Gallen) 1849, Waldau (Bern) 1855(47), Rosegg (Solothurn) 1860, Rheinau (Zürich) 1867(42), Königsfelden (Aargau) 1869, Burghölzli (Zürich) 1870 (4a), Cery (Vaud) 1873 (34), St. Urban (Luzern) 1873, Marsens (Fribourg) 1875, Friedmatt (Basel) 1886, Breitenau (Schaff hausen) 1891, Waldhaus (Graubünden) 1892 (34), Wil (St. Gallen) 1892, Münsingen (Bern) 1895, Perreux (Neuchâtel) 1895, Casvengo (Ticino) 1898, Bel-Air (Genève) 1900. 
Ausländische Experten interessierten sich schon früh für die Reformtätigkeit in unserem Lande, so Joseph Guislain (1840), Giovanni Tonino (1864) und insbesondere L. J. LUNIER (1867/68).

Auffallend war die große Vielgestaltigkeit der Verhältnisse. Unter den zehn Anstalten, die Lunier genauerer Beschreibung für würdig hält, fand er nur drei, nämlich Préfarier, Waldau-Bern und Rosegg-Solothurn wirklich gut, genügend findet er die Genfer Anstalt «Les Vernets», während er die anderen eher als mangelhaft ansieht und diejenigen in Basel und Zürich als für so fortschrittliche Gemeindewesen unwürdig hält. Doch befand man sich mitten in einer aktiven Aufbauperiode, die Pläne für die Anstalten in Königsfelden, Zürich und Lausanne waren schon weit gediehen oder in Ausführung begriffen (25). Baulich wurde zunächst das «Blocksystem» angewandt, d. h. es wurde ein Hauptbau errichtet - bei den nur umgebauten Gebäuden ergab sich das ja ohnehin. In den 1880er Jahren ging man zum «Pavillonsystem» über, d. h. die einzelnen Abteilungen wurden in kleineren Einzelgebäuden untergebracht. Eine tiefgehende Umgestaltung der Anstalt in organisatorischer oder ärztlicher Beziehung war damit aber nicht verbunden. Die Privatanstalten, die sich in diesen Jahrzehnten ebenfalls kräftig entwickelten, organisierten sich nach ähnlichen Prinzipien. Dieser Anstaltstypus ist nun also beiläufig über hundert Jahre alt. Natürlich wurde ständig vieles verbessert und den neuen ärztlichen, namentlich auch therapeutischen Forderungen angepaßt, am Prinzip der Anstaltsführung und der Trennung von den Allgemeinspitälern wurde aber festgehalten, so daß es wohl berechtigt ist, von einer gewissen Erstarrung der praktischen Psychiatrie zu sprechen. Wilhelm Griesinger hatte diese Gefahr erstaunlich früh erkannt (1868), doch vermochte er mit seinen energisch vorgetragenen Forderungen nicht durchzudringen (43).

\section{Anfünge einer Spezialisierung des ärztlichen und pflegerischen Personals}

Da die Psychiatrie sich nur allmählich aus der allgemeinen bzw. der inneren Medizin heraus entwickelte, waren die früheren «Irrenärzte» meist praktische Ärzte oder Internisten. Beim Ausbau der Anstaltspsychiatrie in der Schweiz, die im dritten Viertel des letzten Jahrhunderts wohl ihre größte Intensität erreichte, waren Psychiater aus Deutschland - wo die Spezialisierung weiter fortgeschritten war - maßgeblich beteiligt.

Henri Ellenberger stellt treffend fest: 
«Au point de vue psychiatrique, la Suisse resta assez longtemps une colonie spirituelle de l'Allemagne, qui lui envoyait des professeurs, des aliénistes et du personnel infirmier supérieur» (10).

Freilich wurden die Voraussetzungen für das Wirken dieser Deutschen im Lande selbst geschaffen, ihr Einfluß war aber doch so groß, daß man von einer «deutschen Periode» sprechen kann (43), wie sie übrigens nicht nur auf dem Gebiet der Psychiatrie, sondern in der ganzen Medizin, ja im Geistesleben der allemannischen Schweiz überhaupt festzustellen ist. Es sind hier namentlich folgende Psychiater zu nennen:

Ludwig Binswanger (1820-1860) aus Bayern übernahm 1850 die Direktion der Anstalt Münsterlingen (Thurgau), um aber schon 1857 eine eigene Anstalt «Belle-vue» im nahen Kreuzlingen zu gründen, die noch heute von seinen Nachkommen geführt wird und die aus der Geschichte der Schweizer Psychiatrie nicht wegzudenken ist. Ludwig Binswanger wurde der Stammvater einer ganzen Dynastie von Psychiatern, deren bekanntester sein Enkel Ludwig Binswanger (1881-1966) werden sollte. August Zinn (1825-1897), einer der zahlreichen Flüchtlinge aus Deutschland, hatte am «alten Spital» und am «Irrenhaus» in Zürich gearbeitet und hatte in einer aufrüttelnden Schrift auf die veralteten Zustände des «Zürcher Irrenwesens» hingewiesen. Am folgenreichsten war aber seine Tätigkeit als Direktor der Anstalt St.Pirminsberg (St. Gallen), die er nach seinen Auffassungen neu gestaltete. Er gründete auch den ersten Hilfsverein für Geisteskranke in unserem Land. Im Jahre 1872 kehrte er nach Deutschland zurück, wo er als eine Autorität in psychiatrischen Anstaltsfragen galt und auch politisch tätig war. Heinrich Cramer (1831-1893) war Assistent in St. Pirminsberg, als er 1861 zum Direktor der solothurnischen Anstalt berufen wurde, wo er auf Zwangsmaßnahmen verzichtete und damit zu einem der Pioniere des «no restraint » in unserem Lande wurde. L. Lunier konnte in der Rosegg keine einzige Zwangsjacke in Gebrauch sehen, obwohl er bei seinen Anstaltsbesuchen speziell auf Zwangsmittel achtete. Ludwig WiLLE (1838-1912) kam 1864 in die Schweiz, um die Direktion der Anstalt Münsterlingen zu übernehmen, doch bereits 1867 rief man ihn nach Rheinau (Kanton Zürich), wo man das eben aufgehobene Kloster in eine Pflegeanstalt umgestaltet hatte. 1873 wurde ihm eine ganz ähnliche Aufgabe in St. Urban (Luzern) übertragen. Von hier kam er 1875 als Professor für Psychiatrie nach Basel, wo er zunächst die alte Irrenabteilung am Bürgerspital leitete, um 1886 die neugebaute Klinik Friedmatt zu beziehen, an deren Projek- 
tierung er wesentlich beteiligt war $(42,26)$. Diese deutschen Psychiater brachten die neuesten Prinzipien in unser Land, namentlich das «no restraint», sie bemühten sich um Therapie und Fürsorge und forderten die psychiatrische Ausbiidung aller zukünftigen Ärzte (43).

Ein Zeichen für den sich entwickelnden Stand der Psychiater bildete die Gründung eines - zunächst freilich sehr kleinen - «Vereins der Irrenärzte», die im Jahre 1864 in der bernischen Klinik Waldau stattfand (24). Hauptsächlicher Initiant war Henri Borrel (1812-1872), Leiter der Anstalt Préfargier (Neuchâtel). DieVerhandlungen erreichten bald ein hohes wissenschaftliches Niveau, so wurde etwa die 7. Versammlung in Basel vom Jahre 1871 von Männern wie Bernhard Gudden, Heinrich Laehr, Wilhelm His, Ludwig Wille, Carl Liebermeister, Bernhard Naunyn u. a. besucht (7a).

Die Wichtigkeit eines geschulten Pflegepersonals wurde zwar immer deutlicher erkannt, eine systematische Ausbildung der Pfleger und Pflegerinnen wurde aber erst nach 1900 allmählich eingeführt.

\section{Lehr- und Forschungstätigkeit}

Die Psychiatrie gehörte als akademisches Lehrfach bis weit in das 19. Jahrhundert hinein zur inneren Medizin. Es sei lediglich daran erinnert, daß Philipp Pinrl zu seiner Zeit hauptsächlich als Internist bekannt war und daß Wilhelm Griesinger erst 1865, drei Jahre vor seinem Tode, seine internistische Professur mit einer psychiatrischen vertauschte. An der damals einzigen schweizerischen Universität, in Basel, wurde 1801 JoHANN Јаков SтÜскеLBERger (1758-1838) zu einem regelmäßigen Besuch des Irrenhauses mit seinen Studenten verpflichtet, ob die angekündigte psychiatrische Vorlesung aber zustande kam, ist ungewiß (7). Sogar Friedrich Brenner (1808-1874), Dozent für «Psychiatrie, Pädiatrie und Arzneimittellehre» (!) und ab 1855 a.o. Professor für Psychiatrie, brachte meist keine Vorlesung zustande. Dies änderte sich erst mit der Berufung von Ludwig WiLLe im Jahre 1875; seine Vorlesungen und seine klinischen Visiten waren von Anfang an gut besucht $(45,46)$. Ob J. F. A. Tribolet (1794-1871) in Bern bereits Vorlesungen über Psychiatrie hielt, ist unwahrscheinlich, doch habilitierte sich Rudolf Schärer (1823-1890) im Jahre 1861 für Psychiatrie, und von nun an fanden auch klinische Vorlesungen statt (47). Bei Schärer hat übrigens der junge Eugen Bleuler seine erste klinische Ausbildung genossen. Schärers Nachfolger im Jahre 1890 war 
Wilhelm von Speyr (1852-1939), der als Forel-Schüler bereits einer neuen Epoche angehörte (12). Mittlerweile war, im Jahre 1888, die Psychiatrie in der Schweiz auch Prüfungsfach geworden, womit allen zukünftigen Ärzten wenigstens eine einfache Grundausbildung in Psychiatrie vermittelt wurde. Die Universitätskantone wurden durch die neue Prüfungsordnung gezwungen, mehr für den psychiatrischen Unterricht zu tun: In Lausanne wurde 1889 Siegrried Rabow (1848-1931) zum Professor für Psychiatrie ernannt, in Genf zögerte man noch länger: erst 1896 wurde JoHANnès Martin (1851-1939) Professor für Psychiatrie; er hatte allerdings schon vorher Unterricht erteilt (24a). In Zürich war zunächst ebenfalls nicht viel geschehen. Lucas Schönlein (1793-1864), der erste Internist an der Universitätsklinik (1833-1839), der ja die Psychiatrie zu vertreten hatte, scheint sich für Geisteskranke nicht sehr interessiert zu haben, ebensowenig seine nächsten Nachfolger. Die Leitung der Irrenabteilung überließ man dem allerdings sehr tüchtigen Chr. E. BACH (1810-1873). Mit WiLhelm Griesinger erhielt Zürich aber von 1860 bis 1865 einen Internisten, der zugleich ein hervorragender Psychiater war. Er hielt psychiatrische Vorlesungen, führte an der alten «Irrenanstalt» das «No restraint» ein und war maßgebend an der Planung der neuen psychiatrischen Klinik beteiligt (4). In seinem berühmten und einflußreichen Werk fanden sich ausgedehnte psychologische Erörterungen, die psychischen Ursachen hielt er «für die häufigsten und ergiebigsten Quellen des Irreseins »; der psychischen Behandlung schenkte er große Aufmerksamkeit (16). Griesinger hat trotz seines nur kurzen Aufenthaltes in Zürich die Schweizer Psychiatrie nachhaltig beeinflußt. Vier Jahre nach seinem Wegzug wurde in Zürich eine Professur für Psychiatrie geschaffen (1869), mit welcher Bernhard von Gudden (1824-1886) betraut wurde. Gudden war ein hervorragender Anstaltspsychiater, der schon in seiner früheren Klinik Werneck die Zwangsmaßnahmen abgeschafft hatte, seine wissenschaftlichen Bemühungen galten aber fast ausschließlich der Hirnforschung. Da er nur vier Jahre (18691873) in Zürich tätig war, war sein direkter Einfluß nicht besonders groß, um so größer war seine indirekte Wirkung, indem er den jungen August Forel für die psychiatrische Laufbahn gewann. Wie sehr damals die Hirnforschung im Vordergrund stand, erwies die Berufung von Eduard Hitzig (1838-1907), der durch seine Entdeckung der elektrischen Erregbarkeit der Hirnrinde bekannt geworden war. Ein guter Hirnphysiologe ist aber nicht unbedingt auch ein guter Psychiater, und vielleicht hängt es damit zusammen, daß er mit der Leitung der Anstalt wenig Freude erlebte. Schon 
1879 ging er deshalb nach Halle. Sein Nachfolger wurde August Forel (1848-1931, in Zürich 1879-1898). Mit Forel beginnt eine neue Epoche: Er ist der eigentliche Begründer einer Psychiatrie schweizerischer Prägung.

Aber auch andernorts regten sich die einheimischen Kräfte. Gotтlieb Burckhardt (1836-1907), der sich schon 1863 in Basel für innere Medizin und Nervenkrankheiten habilitiert hatte, dann in Bern wirkte und 1882 Direktor der Anstalt Préfargier wurde, versuchte durch eine besondere Hirnoperation chronische Geisteskrankheiten zu bessern. Dieser Vorläufer der Topektomie eilte der Zeit um Jahrzehnte voraus, so daß sein Verfahren abgelehnt wurde und in Vergessenheit geriet (36). Wie in Deutschland bestand auch bei uns ein verbreitetes Bedürfnis nach psychiatrischen Kenntnissen : 1865 erschien ein Buch von Hugo Henne (1833-1891) Über Geistesstörungen und Irrenanstalten, das der «Aufklärung für Ärzte und gebildete Laien » dienen sollte; unnötig zu sagen, daß Henne sich besonders an Griesingers Lehrbuch anlehnte (19). Die übrigen Schweizer Psychiater dieser Generation konnten sich offenbar nicht zu einer wissenschaftlichen Tätigkeit entschließen, so wesentlich auch ihre praktische Arbeit gewesen sein mag. Zu erwähnen wäre etwa R.F.Fetscherin (1829-1892), Th. Challand (1841-1888), H. Borrel (1812-1872), E. SchaufelbüEL (1831-1902), L. Greppin (1854-1925). Ohne die aktiven deutschen Zuzüge wäre die Schweizer Psychiatrie wohl noch lange provinziell geblieben (43).

\section{Die Anfänge einer Psychiatrie schweizerischer Prägung}

«C'est avec Forel que la psychiatrie suisse prend conscience d'ellemême», schreibt Henry Ellenberger (10); und man kann hinzufügen, daß die Schweizer Psychiatrie nicht nur ihre Eigenart erkannte, sondern auch die Kraft und das Selbstbewußtsein gewann, anders zu sein, und zu ihrer eigenen Auffassung zu stehen. Vielleicht noch deutlicher wird dies bei Forels Schüler und Nachfolger Eugen Bleuler. Mit diesen Männern, die nacheinander fast ein halbes Jahrhundert in Zürich wirkten, war eine Auffassung etabliert, die sich schweizerisch nennen durfte.

August Forel (1848-1931) stammte aus einer großen, alten Waadtländer Familie. Über sein Leben wissen wir durch seine Selbstbiographie und durch einen Briefband (11, 12, 5a), sehr gut Bescheid. Trotz vorwiegend naturwissenschaftlichen Interessen entschlo $\beta$ er sich zum Studium der Medizin. Bereits als Student bereitete er ein großes Werk über die Ameisen der Schweiz vor, welches dem jungen Autor einen Preis der Académie des 
Sciences in Paris und das Lob von Charles Darwin einbrachte. Die Vorlesungen von BERNHARD von GUDDEN zogen ihn besonders an, so daß er am Ende seines Studiums eine Dissertation in Hirnanatomie schrieb, wozu er zu Theodor Meynert nach Wien ging. In den Jahren 1873-1878 war er Assistenzarzt bei Bernhard von Gudden in München; dort habilitierte er sich im Jahre 1877. Zwei Jahre später wurde er Professor für Psychiatrie in Zürich, wo er bis 1898 blieb.

Für Forels Lehrer Gudden ruhte die Psychiatrie auf zwei wirklich sehr verschiedenen Säulen: der Anstaltsführung und der Hirnanatomie. Forel richtete denn auch in Zürich zuerst ein Laboratorium für Hirnanatomie ein. Es gelang ihm bald ein großer Wurf: 1886 formulierte er als einer der ersten die «Neuronenlehre», zu deren Begründer er gehört (2). Er begünstigte die Habilitation des Hirnforschers Constantin von Monakow (1853-1930) an der Zürcher Universität (27). Um diese Zeit wandelten sich aber seine Interessen durch Einbeziehung der Psychologie: Er wandte sich dem Hypnotismus zu, den er 1887 bei H.M.Bernheim und A.A.Liebeault in Nancy studierte, und der ihm tiefe Einsichten in das Leib-Seele-Problem im Sinne einer monistischen Auffassung verschaffte (37). Führende Juristen wie Carl Stoss und Karl von Lilienthal informierten sich bei Forel über den Hypnotismus, der damit für Reformen in der Rechtssprechung Bedeutung erlangte, wie sich Forel überhaupt auch der forensischen Psychiatrie besonders annahm. Im Hypnotismus gewann Forel auch ein wichtiges Werkzeug zur Therapie nervöser Störungen. Er wurde einer der Hauptvertreter des auch im deutschen Sprachgebiet heftig umkämpften Hypnotismus. Sigmund Freud widmete Forels Buch über die Hypnose eine ausführliche Besprechung, mit einem Angriff gegen den gemeinsamen Lehrer Theodor Meynert, der den Hypnotismus ablehnte (15). Mit der hypnotischen Psychotherapie war die Vorbedingung für eine « ambulante Psychiatrie» in Poliklinik und Privatpraxis gegeben. Wenn Forels Schüler - insbesondere Eugen Bleuler und Adolf Meyer - später eine «dynamische Psychiatrie» vertraten, so beruht das auf ihrer Schulung im Hypnotismus. Forel selbst lehnte später die Psychoanalyse Freuds freilich ab. Therapeutisches und dann auch prophylaktisches Denken waren maßgebend für Forels Kampf gegen den Alkoholismus. Er war einer der wichtigsten internationalen Vertreter der Alkoholabstinenz (8). Mit seinen Büchern über die sexuelle Frage und über die Hygiene der Nerven und des Geistes wurde er zu einem Vorläufer der Psychohygiene, die sein Schüler Adolf Meyer in den Vereinigten Staaten besonders wirkungsvoll vertrat (39). 
August Forel schuf eine Psychiatrie schweizerischer Prägung, mit seinen Schülern Eugen Bleuler und Adolf Meyer erlangte sie Weltgeltung. Die Würdigung dieser beiden Psychiater gehört nicht mehr in den Rahmen dieser kurzen Übersicht, denn ihre hauptsächliche Wirksamkeit fällt in die Zeit nach 1900. Es sei hier lediglich noch an die zeitweilige enge wissenschaftliche Verbindung von Eugen Bleuler mit Sigmund Freud erinnert und auch daran, daß Carl Gustav Jung jahrelang ein enger Mitarbeiter Eugen Bleulers war.

Zur Zeit von Pinels Reform bot das «Irrenwesen» in unserem Lande ein geradezu elendigliches Bild. Bis gegen 1850 war die Schweiz in der praktischen Psychiatrie noch sehr rückständig, eine wissenschaftliche Psychiatrie gab es noch nicht. Die wenigen Jahrzehnte bis 1900 genügten, um die Schweizer Psychiatrie in die vordersten Ränge internationaler Geltung vorrücken zu lassen. Wie dieser Prozeß vor sich ging, ist auch über das Gebiet der Psychiatriegeschichte hinaus von Bedeutung.

\section{Literatur}

1 Ackerknecht E.H., Kurze Geschichte der Psychiatrie, 2. Auflage, Stuttgart 1967.

2 Andreoli A., Zur geschichtlichen Entwicklung der Neuronenlehre, Basel/Stuttgart 1961.

3 Bersot H.,Destins de la psychiatrie suisse, Berne, s.d.

4. Bleuler M., Geschichte des Burghölzlis und der psychiatrischen Universitätsklinik, in Zürcher Spitalgeschichte, Zürich 1951, 2. Band (vgl. auch im gleichen Werk die Beiträge von W.LöFFLER und B.MILT).

4a (Herausgeber) Kantonale Psychiatrische Universitätsklinik Burghölzli Zürich 1870 bis 1970, Zürich 1970.

5 Brenner F., Bericht über die Wirksamkeit der Irrenanstalt in Basel, Basel 1872.

5 a Buess H. (Herausgeber), Schweizer Ärzte als Forscher, Entdecker und Erfinder, Basel 1946.

6 Burckhardt A.und F.Brenner, Über die Übelstände in der Basler Irrenanstalt und die Notwendigkeit ihrer Verlegung, Basel 1865.

7 BurcKhardt A., Geschichte der medizinischen Fakultät Basel 1460-1900, Basel 1917.

7a Correspondenz-Blatt für Schweizer Ärzte I (1871) 283 (und die folgenden Jahrgänge!)

8 Debrunner R., Alkoholabstinenz und Psychiatrie im 19. Jahrhundert, Diss. med.,Zürich 1961.

9 EBstein E.,Aus Schönleins psychiatrischer Lehrtätigkeit in Würzburg, Z.ges. Neurol. Psych.38 (1918) 322,328.

10 Ellenberger H., La psychiatrie suisse, Aurillac, s.d. (1953).

11 Forel A., Rückblick auf mein Leben, 2. Auflage, Zürich 194.7. 
12 - Briefe/Correspondance 1864, 1927, herausgegeben von HANS H. WALSER, Bern/ Stuttgart 1968.

- Der Hypnotismus, seine Bedeutung und seine Handhabung, 1. Auflage, Stuttgart 1889.

- Gesammelte hirnanatomische Abhandlungen, München 1907.

15 Freud S., Forel: Der Hypnotismus (usw.), Wiener med. Wschr. 1889, 1098-1100, 1892-1896 (Besprechung von Nr.13).

16 Griesinger W., Die Pathologie und Therapie psychischer Krankheiten, 2.Auflage, Stuttgart 1861.

17 - Zur Kenntnis der heutigen Psychiatrie in Deutschland, Leipzig 1868.

18 Guislain J., Lettre médicales sur l'Italie, avec quelques renseignements sur la Suisse, Gand 1840.

19 Henne H., Über Geistesstörungen und Irrenanstalten. Zur Aufklärung für Ärzte und gebildete Laien, St. Gallen 1865.

20 Herzog R.und H. WALSER, Johann Konrad Fäsi-Gessner (1796-1870), Unbekannte Zeichnungen aus dem alten Zürcher Spital, Zürcher Taschenbuch auf das Jahr 1969, Zürich 1968,S.121-146.

21 Hintzsche E.,600 Jahre Krankenpflege im Berner Inselspital, Bern 1954.

22 Hofmann M.,Die Irrenfürsorge im alten Spital und Irrenhaus Zürich, Zürich 1922.

23 HungerbüHLer J.M., Über das öffentliche Irrenwesen in der Schweiz, St.Gallen/Bern 1846.

24. Ladame Сн., Histoire de la société des médecins aliénistes suisse 1864-1919, Arch. Suisse Neurol.Psych.7 (1920) 157-166,352-357; 9 (1922) 130-141.

24 a - Joannès Martin (1851-1939), Revue médicale de la Suisse Romande 59 (1939) 493-505.

24b Laehr H., Gedenktage der Psychiatrie, 4. Auflage.

25 Lunier L., De l'aliénation mentale en Suisse. Annales médico-psychol.10 (1867) 1-6, 233-262,433-467; 11 (1868) 16-69,169-202,378-402.

26 Meyer Chr., Ludwig Wille 1834-1912, Diss.med.,Zürich (erscheint voraussichtlich 1973).

27 Monakow C.von, Vita mea,Autobiographie, herausgegeben von A.Gubser, Bern/ Stuttgart/Wien 1970.

28 Morgenthaler W., Bernisches Irrenwesen von den Anfängen bis zur Eröffnung des Tollhauses 1794, Bern 1915.

29 Müllener E. R., Die Entstehung des Kleptomaniebegriffes, Sudhoffs Arch.48 (1964) 216-239.

30 Müller Chr., Gottlieb Burckhardt, the father of topectomy, Amer.J. Psych. 117 (1960) $4.61 \mathrm{ff}$.

31 Отто C., Reise durch die Schweiz, Italien, Frankreich, Großbritannien und Holland, Hamburg 1825.

32 Rengger H., Dr.med. J. Guggenbühl, der Begründer der ersten Heilanstalt für Kretinen, Diss. med.,Zürich 1927.

33 Schüler G., Der Basler Irrenarzt Friedrich Brenner (1809-1874), Diss.med.,Zürich (erscheint Ende 1972).

34. Sтеск H., De l'asile d'aliénés à l'hôpital psychiatrique, Lausanne 1948.

35 Tonino G., Sopra alcuni manicomii di Francia e Svizzera con brevi parole sopra altri d'Inghelterra e d'Italia, Torino 1864. 
36 Tribolet A., Bericht über die neue Irren-Heil- und Pflegeanstalt Waldau im Kanton Bern, Bern 1855.

37 Walser H., L'école hypnologique de Nancy, Médicine et Hygiène 23 (28 avril 1965) 443.

38 - Anfänge der wissenschaftlichen Psychiatrie in der Schweiz, in Aktuelle Probleme aus der Geschichte der Medizin (Verhandlungen des XIX. Internationalen Kongresses für Geschichte der Medizin, Basel 1964), Basel/New York 1966, S.477-482.

39 - Die wissenschaftlichen Anfänge von Adolf Meyer (1866-1950) und die Entstehung der «Zürcher psychiatrischen Schule», Gesnerus 23 (1966) 202-210.

40 - Everyday Life in a European Hospital in the $1830 \mathrm{~s}$ and $1840 \mathrm{~s}$, J.Hist.Med. 25 (1970) 22-30.

41 - Der Entschluß zum Bau der Heilanstalt Burghölzli, in Kantonale psychiatrische Universitätsklinik Burghölzli 1870-1970, herausgegeben von M. BLEULER, Zürich 1970, S.11-18.

42 - Hundert Jahre Klinik Rheinau 1867-1967, Aarau 1970 (Veröffentlichung der Schweizerischen Gesellschaft für Geschichte der Medizin und der Naturwissenschaften, XXIV.Band).

43 - Die «deutsche Periode» (etwa 1850-1880) in der Geschichte der Schweizer Psychiatrie, Gesnerus 28 (1971) 47-55.

43 a - Historische Grundlagen der heutigen Psychiatrie in Graubünden, Äskulap in Graubünden, Chur 1970.

44. Wille H., Hundert Jahre Heil- und Pflegeanstalt Münsterlingen, Frauenfeld 1944 (Historischer Verein des Kantons Thurgau, Heft 80).

45 Wille L., Zum «No restraint», Basel 1880.

46 - Úber psychiatrische Klinik, Basel 1882.

47 Wyrsce J.,Hundert Jahre Waldau, Bern/Stuttgart 1955.

48 Zeitschrift, Allgemeine - für Psychiatrie, Berlin, I, 1844ff. (Diese Zeitschrift ist für die Kenntnis der Psychiatrie in der alemannischen Schweiz im 19. Jahrhundert stets beizuziehen).

49 Ziegler M., Die Entwicklung des Irrenwesens in Basel, Diss.med., Basel 1933.

50 Bressler H. G., Königsfelden 1872-1972, Zentenarschrift einer Psychiatrischen Klinik, Aarau [1972] (erschienen während der Drucklegung der vorliegenden Arbeit).

51 Jacky C., Die Einführung des «No restraint» im deutschen Sprachgebiet. Diss. med., Zürich (Manuskript, erscheint 1974).

52 Schenker B., Der «Burghölzliskandal» von 1879 in seiner Bedeutung für das Schweizer Irrenwesen. Diss. med., Zürich (in Vorbereitung). 